\section{Robust Dissipative Compensators for Flexible Spacecraft Control}

S. M. JOSHI, Senior Member, IEEE

P. G. MAGHAMI, Member, IEEE

NASA Langley Research Center

The problem of controller design for flexible spacecraft is addressed. Model-based compensators, which rely on the knowledge of the system parameters to "tune" the state estimator, are first considered, and are shown to have high sensitivity to parameter uncertainties. Three types of dissipative controllers, which use collocated actuators and sensors, are next considered. These controllers guarantee stability in the presence of unmodeled elastic modes and parameter uncertainties. A procedure is given for designing an optimal dissipative dynamic compensator, which can provide better performance while still retaining robust stability

Manuscript received February 15, 1991; revised July 17, 1991. IEEE Log No. 9107188.

Authors' address: NASA Langley Research Center, MS230, Hampton, VA 23665-5225.

U.S. Government work not protected by U.S. copyright.

\section{INTRODUCTION}

Control of large flexible space structures (LFSS) is a challenging problem because of their special dynamic characteristics which include: low, closely spaced structural mode frequencies, very small inherent damping, and the lack of accurate knowledge of the parameters. Controller design approaches for attitude control and vibration suppression of flexible spacecraft have generally evolved into two main classes, namely, model-based controllers (MBCs), and dissipative controllers. MBCs essentially consist of an observer or a state estimator that is "tuned" to a mathematical model ("design" model) of the plant. Such controllers can be typically designed using time-domain techniques (e.g., linear-quadratic-Gaussian (LQG), observer/eigenplacement methods) or frequency-domain (e.g., LQG/loop transfer recovery (LTR) or $\mathrm{H}_{2} / \mathrm{H}_{\infty}$ multivariable loop-shaping) techniques. For practical implementability reasons, the controller has to be of order much lower than the plant. The main problem in control system design is the presence of unmodeled higher-frequency modes, which can cause instability of the closed-loop system. Various methods have been developed over the past decade for designing MBCs which will maintain stability in the presence of unmodeled dynamics (see [1] for a detailed bibliography). However, little attention has been given in the past to the effect of parametric uncertainties. We first review the application of $\mathrm{MBCs}$ designed using multivariable frequency-domain methods. This class of controllers relies on an observer that utilizes the knowledge of the plant in its "prediction" part. A method is given for designing MBCs which provide robustness to unmodeled elastic-mode dynamics. However, such MBCs are generally very sensitive to parameter uncertainties in the modeled part of the elastic-mode dynamics. We investigate the mechanism which causes such high sensitivity and makes this method unsuitable.

The second category of controllers, i.e., dissipative controllers, use collocated and compatible actuators and sensors (e.g., attitude and rate sensors located near torque actuators). They utilize special (passivity-type) input/output characteristics of the plant and offer robust stability in the presence of unmodeled elastic mode dynamics and parameter errors, i.e., they do not depend on the accuracy of the model for ensuring closed-loop stability. For accomplishing attitude fine-pointing and vibration suppression, it is necessary to control both the zero-frequency rigid-body modes, and the elastic modes. A number of papers (for example, see $[2,3]$ ) on dissipative controller design have focused on controlling only the elastic modes, with the implicit assumption that the rigid-body modes can be controlled separately. However, this assumption is not valid because rigid modes are coupled with the elastic modes 
through control inputs and sensor measurements, and cannot be separated from the elastic modes for controller design. We address the problem of designing dissipative controllers for accomplishing both vibration suppression and rigid-body attitude control. A summary of the stability robustness properties of constant-gain dissipative controllers in the presence of unmodeled elastic mode dynamics, parameter uncertainties, actuator/sensor nonlinearities, and first-order actuator dynamics, is presented. A class of robust dynamic dissipative controllers is also discussed. For the single-input/ single-output (SISO) case, we give sufficient conditions for robust closed-loop stability using a dynamic dissipative compensator, and a generalized proportional-plus-derivative compensator, which is a special case of the former. We show that the robust stability properties are retained in the presence of second-order actuator dynamics if acceleration feedback is used. We also propose a two-level dissipative controller which consists of low- and high-authority controllers, and address the problem of obtaining optimal performance combined with robust stability.

\section{A. Mathematical Model}

The linearized mathematical model of a flexible space structure is given by [1]

$$
\bar{A} \ddot{p}+\ddot{B} \dot{p}+\bar{C} p=\Gamma^{T} u
$$

where

$$
p=\left(\alpha^{T}, q^{T}\right)^{T} ; \quad \alpha=(\phi, \theta, \psi)^{T}
$$

where $\alpha$ represents the rigid-body attitude vector, $q$ is the $n_{q} \times 1$ vector of elastic modal amplitudes, and $u$ is the $m \times 1$ input torque vector. $\bar{A}=\tilde{A}^{T}>0$ is the generalized inertia matrix.

$$
\tilde{B}=\operatorname{diag}\left(0_{3}, D\right)
$$

where $0_{3}$ represents the $3 \times 3$ null matrix, and $D=$ $D^{T}>0$ is the $n_{q} \times n_{q}$ matrix representing the inherent damping.

$$
\bar{C}=\operatorname{diag}\left(0_{3}, \Lambda\right)
$$

where

$$
\Lambda=\operatorname{diag}\left(\omega_{1}^{2}, \omega_{2}^{2}, \ldots, \omega_{n q}^{2}\right)
$$

where $\omega_{i}\left(i=1,2, \ldots, n_{q}\right)$ represent the elastic mode frequencies. The attitude and attitude-rate sensor outputs are given by

$$
\begin{gathered}
y_{p}=\Delta_{p} p+w_{p} \\
y_{r}=\Delta_{r} \dot{p}+w_{r}
\end{gathered}
$$

where $y_{p}$ and $y_{r}$ are $l_{1} \times 1$ vectors, $\Delta_{p}, \Delta_{r}$ are $l_{1} \times n_{1}$ matrices $\left(n_{1}=n_{q}+3\right)$, and $w_{p}, w_{r}$ are $l_{1}$-vector zero-mean mutually uncorrelated white noise vectors with covariance intensities $W_{p}$ and $W_{r}$.

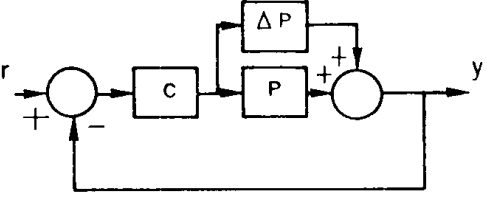

Fig. 1. Additive uncertainty formulation.

By defining the state vector:

$$
x=\left(p^{T}, \dot{p}^{T}\right)^{T}
$$

the equations of motion can be written as

$$
\begin{aligned}
& \dot{x}=A x+B u \\
& y=C x+w
\end{aligned}
$$

where $x$ is the $n$-dimensional state vector $\left(n=2 n_{1}\right)$, and $y$ and $w$ are $l$-vectors $\left(l=2 l_{1}\right)$ :

$$
y=\left(y_{p}^{T}, y_{r}^{T}\right)^{T} ; \quad w=\left(w_{p}^{T}, w_{r}^{T}\right)^{T} .
$$

The main problem in controller design is that the number of elastic modes in the "truth" or "evaluation" model is very large. To design an implementable controller, it is necessary to use a reduced-order "design" model, i.e., $n_{q}$ is much less than the number of elastic modes in the evaluation model.

\section{MODEL BASED COMPENSATORS}

In the case of flexible spacecraft, the plant has an inherently parallel structure; therefore, the unmodeled elastic modes can be lumped into an "additive uncertainty" $\Delta P$ (Fig. 1), so that the plant transfer function matrix (from torque inputs to attitude outputs) is given by

$$
P^{\prime}(s)=P(s)+\Delta P(s)
$$

where $P(s)$ is the design model transfer matrix, consisting of the three rigid rotational modes and a few elastic modes. A sufficient condition for stability in the presence of unmodeled elastic modes is $[4$, p. 273]

$$
\begin{array}{r}
\bar{\sigma}[\Delta P(j \omega)]<\frac{1}{\bar{\sigma}\left[C(j \omega)\{I+P(j \omega) C(j \omega)\}^{-1}\right]} \\
\text { for } 0 \leq \omega<\infty
\end{array}
$$

where $\bar{\sigma}[\cdot]$ denotes the largest singular value, and $C(s)$ is the compensator transfer function matrix. The performance in the frequency domain setting is defined in terms of the bandwidth $\omega_{b}$, which is the frequency above which the "closed-loop gain" $\underline{\sigma}\left(G_{\mathrm{CL}}\right)$ [where $G_{\mathrm{CL}}=P C(I+P C)^{-1}$ and $\underline{g}(\cdot)$ denotes the smallest singular value] starts to roll off below 0.707 (Fig. 2).

As stated previously, the uncertainty $\Delta P$ consists of the contributions of the unmodeled modes. The uncertainty in the frequencies and damping ratios of the unmodeled modes can be represented by lateral and vertical shifts, respectively, of the peaks of $\bar{\sigma}(\Delta P)$. The uncertainty in the mode shapes can 


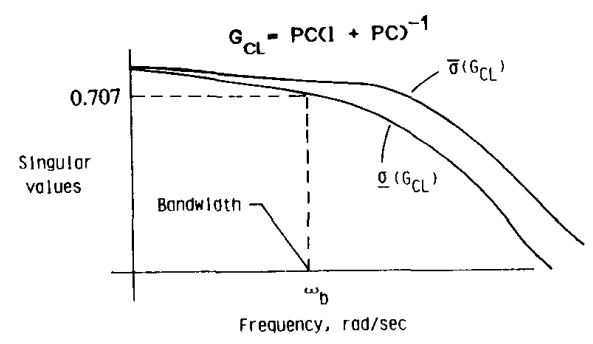

Fig. 2. Definition of bandwidth.

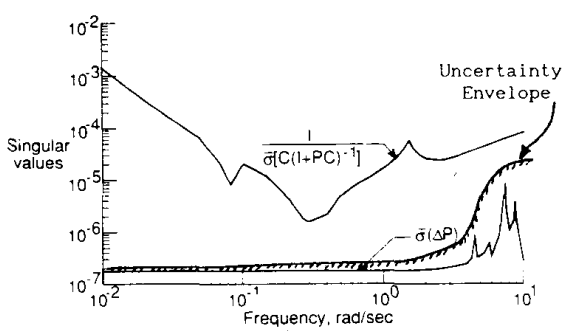

Fig. 3. Frequency-domain robustness condition.

be represented by shifting up the entire $\bar{\sigma}(\Delta P)$ curve. Thus, the uncertainty in the unmodeled dynamics can be represented by an envelope in the $\sigma$-plot of $\Delta P$ (Fig. 3).

The multivariable loop-shaping problem is to obtain a realizable compensator $C(s)$ such that the bandwidth specification is satisfied while simultaneously meeting the robustness condition (12). One method of accomplishing this is the LQG/LTR method [5] which essentially consists of the following steps.

Step 1 Define a "target" (desired) loop gain (indicated by $\sigma[P C]$ ) based on full state feedback design. With the loop broken at the output, the target loop is based on the Kalman-Bucy filter (KBF).

Step 2 "Recover" the target loop gain using an LQG controller by adjusting the weighting matrices for the $L Q$ regulator (LQR).

The target loop is usually designed to satisfy both the performance (bandwidth) specification and robustness condition (12). When the LQG/LTR method was applied to realistic models of flexible spacecraft $[1,6]$, it was found that designing the target loop (i.e., $\mathrm{KBF}$ ) in this manner yields a very conservative controller. A better method is to design the target loop only for performance and to iterate on the LQR weighting matrices to satisfy the robustness condition (12). It is usually necessary to iterate on both the target loop (i.e., KBF) and the LQR to obtain a satisfactory design. It was also apparent that asymptotic loop transfer recovery could be obtained only in the lowest frequency region. However, good loop shaping for performance and robustness could be obtained by iteratively adjusting the LQR and $\mathrm{KBF}$ weighting matrices [1]. This technique provides robustness to unmodeled elastic mode dynamics and uncertainties therein. However, it turns out that the control system is very sensitive to inaccuracies in the design model parameters, in particular, to elastic-mode-frequencies. (This was pointed out in a private communication by Prof. Arthur E. Bryson, Jr. of Stanford University. The high sensitivity of standard LQG controllers was also discussed in [7]). To see this more clearly, consider the SISO case wherein the elastic mode with frequency $\omega_{0}$ and damping ratio $\rho$ is included in the design model. Suppose there is a small error $\Delta \omega$ in the knowledge of $\omega_{0}$, so that the frequency used in the design is $\omega_{1}=\omega_{0}+\Delta \omega$. Then the modeling error due to incorrect knowledge of $\omega_{0}$ is

$$
\Delta(s)=k\left[\frac{1}{s^{2}+2 \rho \omega_{0} s+\omega_{0}^{2}}-\frac{1}{s^{2}+2 \rho \omega_{1} s+\omega_{1}^{2}}\right]
$$

where $k$ is the product of the mode shapes at the actuator and sensor locations. Assuming $\Delta \omega / \omega_{0}=p$ to be small $(\ll 1)$ it can be shown after simple calculation that, for $s=j \omega_{0}$,

$$
\left|\Delta\left(j \omega_{0}\right)\right| \cong \frac{k}{2 \rho \omega_{0}^{2} \sqrt{1+(\rho / p)^{2}}} .
$$

The contribution to $P(s)$ of the elastic mode at $s=$ $j \omega_{0}$ is given by the peak:

$$
\left|P_{0}\left(j \omega_{0}\right)\right|=\frac{k}{2 \rho \omega_{0}^{2}}
$$

so that

$$
\left|\frac{\Delta\left(j \omega_{0}\right)}{P_{0}\left(j \omega_{0}\right)}\right| \cong \frac{1}{\sqrt{1+(\rho / p)^{2}}}
$$

The typical range of $\rho$ is $0.001 \leq \rho \leq 0.01$. If $\rho=0.005$ and $p=0.01$ (i.e., 1 percent error in the knowledge of $\left.\omega_{0}\right)$, we have

$$
\left|\frac{\Delta\left(j \omega_{0}\right)}{P_{0}\left(j \omega_{0}\right)}\right|=0.89
$$

That is, for only 1 percent error in the knowledge of $\omega_{0}$, we get a peak in the modeling error almost as large as the entire contribution of that mode! Thus, having a small error in the knowledge of a design model frequency is as undesirable as not including that mode in the design model! This somewhat surprising phenomenon occurs because of the sharpness of the peaks (i.e., small inherent damping). This additional high peak in the uncertainty envelope would usually cause violation of the robustness condition (12). Extensive numerical studies of iterative frequency-domain observer-based designs showed that this problem occurs for any compensator (not necessarily $L Q G$ or $L T R$ ) wherein the observer uses the knowledge of the design model frequencies. This indicates that, unless the frequencies of the elastic modes in the design model are known very accurately, it is better to avoid their use in a model-based design. 
However, using only rigid-body modes in the design model generally gives very poor performance [1]. One possible method of reducing the sensitivity is to reduce the peaks of the design model by using a low-authority collocated rate feedback controller for damping enhancement [1] as an "inner loop". However, this would couple all the elastic modes and the system would no longer be amenable to additive uncertainty formulation. Another approach is the "maximum entropy, optimal projection method" [8], wherein parameter uncertainties are modeled as multiplicative white noise, and a quadratic performance function is minimized over the parameters of a fixed-order controller. For this method, it would still be necessary to ensure the performance (i.e., bandwidth) and robustness margins, perhaps using the frequency-domain, singular-value setting. In addition, such controllers may introduce high order notch filters (in order to obtain wider notches for accommodating frequency uncertainties) to attenuate the uncertain elastic modes. This may leave the elastic modes unaffected rather than controlling them.

\section{DISSIPATIVE CONTROLLERS}

In view of the sensitivity problem associated with $\mathrm{MBCs}$, the alternative provided by dissipative controllers seems very attractive. Dissipative controllers basically use collocated compatible actuators and sensors (i.e., torque actuators and angular position and rate sensors), so that [see (6) and (7)]

$$
\Delta_{p}=\Delta_{r}=\Gamma \text {. }
$$

The constant-gain dissipative control law is given by

$$
u=-G_{p} y_{p}-G_{r} y_{r}
$$

where $G_{p}$ and $G_{r}$ are symmetric, positive-definite, proportional and rate gain matrices. It has been shown [1] that this control law gives guaranteed asymptotic stability regardless of unmodeled elastic modes or parameter uncertainties. (It was also shown in [1] that stability is maintained even if small imprecision exists in the collocation of the actuators and sensors). Furthermore, if $G_{p}$ and $G_{r}$ are diagonal, this robust stability property is carried over in the presence of: 1 ) monotonically increasing actuator nonlinearities, rate sensor nonlinearities belonging to the $[0, \infty)$-sector, and position sensor nonlinearities belonging to the $(0, \infty)$-sector. (Please note that a single-valued function $\psi(\nu)$ is said to belong to the $(0, \infty)$ sector if $\nu \psi(\nu) \geq 0$ and $\nu \psi(\nu)>0$ for $\nu \neq 0 ; \psi$ is said to belong to the $[0, \infty)$ sector if $\nu \psi(\nu) \geq 0$ (Fig. 4)), and 2) stable actuator dynamics $g_{a}(s)=k /(s+a)$, provided that $g_{p} / g_{r}<a$, where $g_{p}$ and $g_{r}$ denote the appropriate diagonal elements of $G_{p}$ and $G_{r}$. The drawback of these controllers is that the performance is inherently limited because of the structure of the controller.

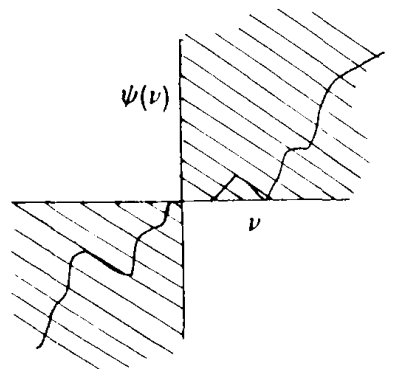

Fig. 4. Nonlinearity belonging to $[0, \infty)$ sector.

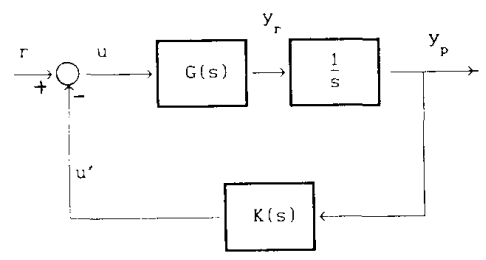

Fig. 5. Dissipative dynamic compensator.

$G_{p}$ and $G_{r}$ can be designed to minimize a quadratic performance function, or to obtain closed-loop eigenvalues close to the desired locations in the least-square sense [1].

\section{A. Dissipative Dynamic Compensators}

In order to obtain better performance while still retaining the guaranteed robustness to unmodeled dynamics and parameter uncertainties, we consider a class of dynamic dissipative controllers. For the SISO case, the plant with the angular position $\left(y_{p}\right)$ as the output is given by Fig. 5, where

$$
G(s)=\frac{1}{J_{s}}+\sum \frac{\phi_{i}^{2} s}{s^{2}+2 \rho_{i} \omega_{i} s+\omega_{i}^{2}}
$$

wherein $J$ is the moment of inertia, and $\phi_{i}, \rho_{i}$, and $\omega_{i}$ denote the rotational mode shape, damping ratio, and natural frequency of the $i$ th structural mode. The transfer function $G(s)$ is positive-real (PR) as defined below [9].

DEFINITION: A rational function $G(s)$ of the complex variable $s$ is positive-real if $G(s)$ is real when $s$ is real, and $\operatorname{Re}[G(s)] \geq 0$ for $\operatorname{Re}[s] \geq 0$.

$P R$ functions have a relative degree (i.e., the difference between the degrees of the denominator and numerator polynomials) of $-1,0$, or 1 . It can also be shown that PR functions have no zeros or poles in the open right-half of the complex plane, and that the poles on the imaginary axis are simple and have nonnegative residues.

It can be seen that, although the transfer function from $u$ to $y_{p}$ is not PR, $G(s)$ is PR. Consider the closed-loop system in Fig. 5 with " $y_{r}$ " as the 
output, where the compensator is given by $C(s)=$ $K(s) / s$. Suppose $K(s)$ is proper and has no unstable cancellations. Using the fact that $G(s)$ is PR, the following result can be obtained.

THEOREM 1 The closed-loop system in Fig. 5 (with $r \equiv 0)$ is asymptotically stable (AS) if a) $K(s)$ has no zeros at the origin, and b) $\operatorname{Re}[K(j \omega) /(j \omega)]>0$ for all real $\omega$.

Outline OF Proof. From [10, Lemma 1], $C(s)=$ $K(s) / s$ stabilizes $G(s)$; i.e., if $C$ and $P$ are represented by stabilizable and detectable realizations, the closed-loop system of Fig. 5 (with $r \equiv 0$ ) is AS. $C(s)$ can be realized by the stabilizable and detectable realization:

$$
\begin{aligned}
\dot{x}_{k} & =A_{k} x_{k}+B_{k} y_{r} \\
\dot{y}_{p} & =y_{r} \\
u^{\prime} & =C_{k} x_{k}+C_{p} y_{p}+D y_{r}
\end{aligned}
$$

Therefore the closed-loop system is AS. ${ }^{1}$

Theorem 1 can be applied to investigate stability of the generalized proportional-plus-derivative compensator, wherein $G_{p}$ and $G_{r}$ are allowed to be dynamic systems represented by transfer functions $G_{p}(s)$ and $G_{r}(s)$ rather than constant gains.

COROLLARY. For the case where $C(s)=G_{r}(s)+$ $G_{p}(s) / s$, the closed-loop system is $A S$ if $G_{p}, G_{r}$ are stable and proper, $G_{p}$ has no zeros at the origin; $G_{r}$, $G_{p} / s$ are $P R ;$ and $\operatorname{Re}\left[G_{r}(j \omega)\right]>0$ for all real $\omega$.

Consider the case where $G_{p}$ and $G_{r}$ are constant, and stable first-order actuator dynamics represented by the transfer function $g_{a}(s)$ are present where

$$
g_{a}(s)=\frac{k}{s+a} .
$$

Including the actuator dynamics with the compensator, we have

$$
C(s)=\frac{K(s)}{s}=\frac{k\left(G_{p}+s G_{r}\right)}{s(s+a)} .
$$

It can be be shown that $\operatorname{Re}[C(j \omega)]>0$ for all real $\omega$ if $G_{p} / G_{r}<a$. Therefore, from Theorem 1 , the closed-loop system is AS if $G_{p} / G_{r}<a$. This result was proved in [1] (for the multivariable case) using function space methods. The robust stability holds regardless of unmodeled elastic modes or parameter uncertainty.

From practical considerations, it is more realistic to consider second-order actuator dynamics. If the actuator dynamics are second order, it would be impossible to satisfy the condition: $\operatorname{Re}[K(s) / s]>0$

1Please note that we have not used the concept of "strictly" PR systems, which is more restrictive, and which has several nonequivalent definitions. Formal extension of this result to the multivariable case is presently under development. for any proper $G_{p}(s)$ and $G_{r}(s)$. However, if the acceleration measurement is available, let

$$
u=-\left[G_{p} y_{p}+G_{r} y_{r}+G_{a} y_{a}\right]
$$

where $y_{a}$ is the angular acceleration:

$$
y_{a}=\Gamma \ddot{x} .
$$

For this case, suppose we have second-order actuator dynamics:

$$
H(s)=\frac{\beta}{s^{2}+\alpha s+\beta}
$$

where $\alpha>0, b>0$. Then we have

$$
\frac{K(s)}{s}=\frac{\beta\left(s^{2} G_{a}+s G_{r}+G_{p}\right)}{s\left(s^{2}+\alpha s+\beta\right)} .
$$

It can be easily shown that $\operatorname{Re}[K(j \omega) /(j \omega)]>0$ for all real $\omega$ if

$$
G_{r} / G_{a} \leq \alpha<\beta G_{r} / G_{p} .
$$

Thus, the system is AS in the presence of second-order actuator dynamics if the gains are chosen to satisfy (26). Given any positive $\alpha$ and $\beta$, it is always possible to select the gains so that $(26)$ is satisfied. This stability property holds regardless of unmodeled elastic modes or parameter errors.

Extension of these reults to the multivariable case is under development.

\section{B. Two-Level Dissipative Compensators}

Another approach to the design of dissipative dynamic compensators is to use a low-authority constant-gain dissipative controller to stabilize the plant, and then to design an additional high-authority (outer-loop) dynamic dissipative controller to optimize the performance. Such a controller would maintain robust stability in the presence of unmodeled elastic modes and parameter uncertainties.

Considering the case where a constant-gain dissipative controller is already in the loop, the closed-loop equations are given by

$$
\bar{A} \ddot{p}+\bar{B} \dot{p}+\bar{C} p=\Gamma^{T} r
$$

where

$$
\bar{B}=B+\Gamma^{T} G_{p} \Gamma ; \quad \bar{C}=C+\Gamma^{T} G_{r} \Gamma .
$$

It can be shown [1] that $\bar{B}$ and $\bar{C}$ are positive definite and that the system in (27) (with $r \equiv 0$ ) is AS. Using $y_{r}$ as the output of this "inner-loop" system, a dynamic PR high-authority compensator can be inserted in the outer loop which will retain the closed-loop stability. Suppose an $n_{c}$ th order compensator is given by

$$
\begin{aligned}
\dot{z} & =A_{z} z+B_{z} y_{r} \\
r & =-C_{z} z
\end{aligned}
$$


where $A_{z}, B_{z}, C_{z}$ are $n_{c} \times n_{c}, n_{c} \times m$, and $m \times n_{c}$ matrices satisfying the following conditions:

$$
\begin{aligned}
& A_{z}^{T} P_{z}+P_{z} A_{z}=-Q_{z} \\
& C_{z}=B_{z}^{T} P_{z}
\end{aligned}
$$

where $Q_{z}, P_{z}$ are symmetric positive definite matrices. Under these conditions, the compensator is stable and PR [11], and we have the following result.

THEOREM 2 The system given by (27)-(32) is AS.

ProOF. Let

$$
V(p, \dot{p}, z)=p^{T} \bar{C} p+\dot{p}^{T} \tilde{A} \dot{p}+z^{T} P_{z} z
$$

Then it can be shown that

$$
\dot{V}=-\dot{p}^{T} \bar{B} \dot{p}-z^{T} Q_{z} z
$$

Thus $\dot{V} \leq 0$, and $\dot{V} \equiv 0$ only if $\dot{p} \equiv 0$ and $z \equiv 0$, which implies (from (27)) that $p \equiv 0$. Thus, the system is AS.

The complete compensator transfer function is given by

$$
C(s)=\frac{G_{p}}{s}+G_{r}+C_{z}\left(s I-A_{z}\right)^{-1} B_{z} .
$$

There is no restriction on the order $n_{c}$ of the compensator; the robust stability property holds for any $n_{c}>0$. The compensator in (35) has a relative degree of zero, and it can be shown to satisfy the sufficient condition of Theorem 1 for the SISO case. However, it does not appear to be straightforward enough at this point to establish a more general connection between the above result and that in Theorem 1 for the SISO case, wherein a relative degree of " 1 " was allowed.

\section{Optimality of High-Authority Compensator}

The compensator given by (35) guarantees closed-loop stability in the presence of unmodeled elastic modes and parameter uncertainty. It would be highly desirable to have such robust compensators which also provide optimal performance. The following result shows that the "outer loop" compensator can be designed to minimize a certain LQG performance function.

Defining the state vector $x$ as in (8), the state-space model of the system in (27) is given by

$$
\begin{aligned}
\dot{x} & =A x+B r \\
y_{r} & =C x .
\end{aligned}
$$

Consider a high-authority compensator given by (29), (30) where the order of $z$ is the same as that of the design model (i.e., $n_{c}=n$ ). It can be shown that the following relations hold:

$$
\begin{aligned}
A^{T} P+P A & =-\hat{Q} \\
C & =B^{T} P
\end{aligned}
$$

where

$$
P=\left[\begin{array}{cc}
\bar{C} & 0 \\
0 & \tilde{A}
\end{array}\right] ; \quad \hat{Q}=\left[\begin{array}{cc}
0 & 0 \\
0 & \bar{B}
\end{array}\right] .
$$

Suppose $P$ is factored as

$$
P=\Delta^{T} \Delta
$$

where $\Delta$ is an $n \times n$ nonsingular matrix. Then we have the following result

THEOREM 3 The compensator given by (29)-(32) minimizes the performance function:

$$
J=\lim _{t_{f} \rightarrow \infty} \frac{1}{t_{f}} \mathcal{E} \int_{0}^{t_{f}}\left(x^{T} Q x+r^{T} R r\right) d t
$$

where

$$
Q=P B R^{-1} B^{T} P+Q_{1} \quad\left(Q_{1}=Q_{1}^{T}>0\right)
$$

and

$$
Q_{z}=\Delta^{-T} Q_{1} \Delta^{-1}
$$

subject to the constraints:

$$
\begin{aligned}
& \dot{x}=A x+B r+v \\
& y=C x+w
\end{aligned}
$$

where $v$ and $w$ are zero-mean white noise processes with covariance intensities $V$ and $W$, given by

$$
\begin{aligned}
& V=P^{-1} \hat{Q} P^{-1}+B R^{-1} B^{T} \\
& W=R
\end{aligned}
$$

PROOF. The proof can be obtained by incorporating the transformation in [12, Appendix] into [12, Theorem 1].

This result basically provides a method for designing an optimal dissipative high-authority controller. The free parameters in the design of this LQG-type dissipative controller are $R$ and $Q_{1}$ (with $G_{p}$ and $G_{r}$ fixed). Such controllers have the potential to yield superior performance with guaranteed robust stability. The best overall performance can be obtained by using $G_{p}$ and $G_{r}$ as additional design variables.

\section{CONCLUDING REMARKS}

The instability mechanisms in MBCs caused by high sensitivity to the parameters, were investigated. In view of this problem, dissipative controllers, which guarantee robust stability in the presence of unmodeled modes and parameter uncertainty, offer an attractive alternative. Robustness properties of constant-gain dissipative controllers were summarized, and a class of dynamic dissipative controllers was presented. It was shown for the SISO case that the robust stability can be maintained in the presence of second-order actuator dynamics if acceleration feedback is also used. A class of 
dissipative compensators, consisting of a constant-gain low-authority controller and a dynamic high-authority controller, was presented. A procedure was presented for designing a dissipative compensator which is also optimal in the LQG sense. These results would be useful for designing compensators which combine high performance with robust stability.

\section{REFERENCES}

[1] Joshi, S. M. (1989) Control of Large Flexible Space Structures. Berlin: Springer-Verlag, 1989.

[2] Benhabib, R. J., Iwens, R. P., and Jackson, R. L. (1981) Stability of large space structure control systems using positivity concepts.

ALAA Journal of Guidance and Control, 4, 5 (Sept.-Oct. 1981).

[3] McLaren, M. D., and Slater, G. L. (1987)

Robust multivariable control of large space structures using positivity.

ALAA Journal of Guidance, Control, and Dynamics, 10 (July-Aug. 1987).

[4] Vidyasagar, M. (1985)

Control Systems Synthesis: A Factorization Approach. Cambridge, MA: M.I.T. Press, 1985.

[5] Athans, M. (1986)

A tutorial on the LQG/LTR method.

In Proceedings of the 1986 American Control Conference. Seattle, WA, June 1986.
[6] Joshi, S. M., Rowell, L. F., and Armstrong, E. S. (1988) Controller synthesis for large flexible space structures. In Proceedings of the 1988 ASME Winter Annual Meeting, Chicago, Nov. 1988.

[7] Bernstein, D. S., et al. (1985) Numerical solution of the optimal projection/ maximum entropy design equations for low-onder, robust controller design.

Proceedings of the 24th IEEE Conference on Decision \& Control, Ft. Lauderdale, FL, Dec. 1985.

[8] Bernstein, D. S., and Hyland, D. C. (1985)

Optimal projection/maximum entropy approach to designing low-order, robust controllers for flexible space structures.

In Proceedings of the IEEE Conference on Decision and Control, Ft. Lauderdale, FL, Dec. 1985.

[9] Kim, W. H., and Meadows, H. E. (1971) Modern Network Synthesis. New York: Wiley, 1971.

[10] Joshi, S. M. (1991)

Generalized proportional-plus-derivative compensators for a class of uncertain plants. ALAA Journal of Guidance, Control and Dynamics, 14, 3 (May-June 1991).

[11] Anderson, B. D. O. (1967)

A system theory criterion for positive-real matrices. SLAM Journal of Control, 5 (1967).

[12] Lozano-Leal, R., and Joshi, S. M. (1988)

On the design of dissipative LQG-type controllers. In Proceedings of the 27th IEEE Conference on Decision and Control, Austin, TX, Dec. 1988.
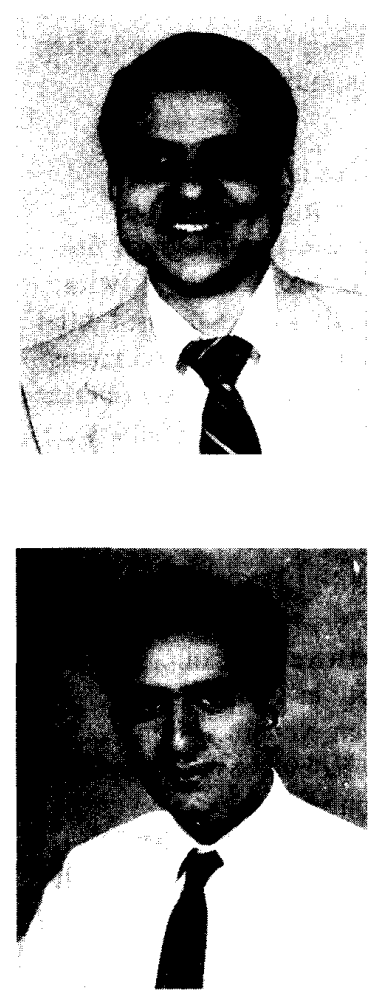

Suresh M. Joshi (S'69-M'73-SM'85) received the Ph.D. degree in electrical engineering from Rensselaer Polytechnic Institute, Troy, NY, in 1973.

Since 1973, he has been at NASA Langley Research Center, Hampton, VA, where he is presently a Senior Research Scientist. He has been performing research on various aspects of control theory and applications to aerospace systems, in particular, to large space structures. He has also served as an adjunct or research faculty member of Old Dominion University, George Washington University, and Pennsylvania State University.

Dr. Joshi is listed in several Who's Who publications, including Who's Who in America, American Men and Women of Science, and Who's Who in Aviation and Aerospace. He is the author of the book Control of Large Flexible Space Structures (Berlin: Springer-Verlag, 1989), and the author or co-author of nearly 120 papers. He is a Senior Member of the IEEE, and has been an elected member of the IEEE-Control Systems Society's Board of Governors since 1989. He is presently an Associate Editor of the IEEE Control Systems Magazine. He is also an Associate Fellow of the American Institute of Aeronautics and Astronautics and a member of the American Society of Mechanical Engineers.

Peiman G. Maghami (M'92) received his B.S., M.S., and D.Sc. degrees from the George Washington University, Washington, DC, in 1980, 1982, and 1984, respectively.

From 1984 to 1985, he worked as a postdoctoral Research Assistant Professor at George Washington University. During 1985-1987, he was a National Research Council Research Associate at NASA Langley Research Center, Hampton, VA. For the period of 1987 to 1989, he was with the Old Dominion University Research Foundation in Norfolk, VA as a Research Assistant Professor, mainly working on grants from NASA Langley Research Center. Since May 1989, he has been with the Spacecraft Controls Branch at NASA Langley Research Center as an aerospace research technologist. His research areas of interest include optimal control of flexible space structures, integrated controls-structures design, control of multibody systems, and stochastic control. $\mathrm{He}$ is a member of American Institute of Aeronautics and Astronautics. 